\title{
Parameter Estimation for Coupled Tank using Estimate Filtering
}

\author{
Jihoon Seung ${ }^{1}$, Amir Atiya F. ${ }^{2}$, Alexander G. Parlos ${ }^{3}$ and Kilto Chong ${ }^{1,4 *}$ \\ ${ }^{1}$ Division of Electronics Engineering, Chonbuk National University, South Korea \\ ${ }^{2}$ Department of Electronics Engineering, Calro University, Egypt \\ ${ }^{3}$ Department of Mechanical Engineering, Texas A\&M University, USA \\ ${ }^{4}$ Advanced Research Center for Electronics and information, JBNU, Jeonju, South \\ Korea \\ seungjihoon@gmail.com,amir@alumni.caltech.edu,aparlos@tamu.edu, \\ *kitchong@jbnu.ac.k
}

\begin{abstract}
In this paper, we present a method of determining the parameters of a dynamic system using state estimate filter. State estimate filters such as Extended Kalman filter and the Unscented Kalman filter are widely used to estimate the status in robot and GPS navigation systems. However, in dynamic systems, determining parameters is difficult because many kinds of the dynamic systems have the properties like a non-linearity and complexity. The work we have done is that the status and parameters are simultaneously estimated to decide the parameter value based on the measurement data set of the system. The works were applied to a coupled tank. The mentioned system was modeled and then setup for computer simulations and experimental purposes to test the performance of the estimation process.
\end{abstract}

Keywords: Parameter Estimation, Extended Kalman Filter, Unscented Kalman Filter, Coupled Tank

\section{Introduction}

Most of the mechanical and electrical systems currently used in industry can be represented as second-order dynamics model comprised of mass, damper, spring, inductances, resistors and capacitors for convenience and ease of control. However, the parameters of a real system have measurement noise due to the difficulties of physical measurement. A change in parameter values can also happen due to the deterioration of the system and sudden change of the environment. It is for these reasons that we determine the optimal parameters of the system using filters such as the extended kalman filter (EKF) or the unscented kalman filter (UKF). Adaptive kalman filters is used for localization algorithms [1,2]. Some traditional methods used in the status estimation of a system are the least squares method (LSM) and maximum likelihood (MLH) among others. The filters have the common property if being applicable to non-linear systems with their own set of advantages and disadvantages. Therefore it is proposed to find which one is suitable for the parameter estimation of nonlinear systems.

In the 1980's, parameter estimation on LSM according to the variation was actively studied by D. T. Lee [3] where it has the disadvantage of the estimate error being repeated when the initial estimation value includes a large error.

${ }^{*}$ Corresponding Author 
Afterward, Jan Swever [4] determined the parameters by applying MLH to decrease measurement noise. Moreover, the parameters were estimated by S. K. Oh [5] using Fuzzy Logic and Neural Network respectively. However, the previous given methods are sensitive to measurement noise.

Park [6] proposed using a modified kalman filter for an adaptive frequency domain channel estimation in orthogonal frequency division multiplexing systems. The state and parameter estimation method on EKF in the dynamic system was proposed by Saadettin Aksoy [7], V. Panuska [8] and Emmanuel Blanchard [9]. Min Zheng [10] performed the method of applying UKF in dynamic system but did so by analyzing the result of a computer simulation and not through an actual experiment.

In order to improve on the enumerated disadvantage, there is a need for a method that not only estimates the state and parameter but is also robust to noise measurements in a real dynamic system.

The EKF and UKF are used to analyze the results of each parameter estimate performance. UKF does not require the linearization process for a state equation by using UT. Therefore the parameter value can be optimally estimated by estimating the optimal state and also running the covariance update process recursively.

A coupled tank was used for the experimental tests. It was constructed with a NI DAQ board and simulated in MATLAB to apply the estimate filters on a real system. Computer simulation and experiments for parameter estimation were performed using the dynamic equation derived for the system. The results were analyzed with various implementations of EKF and UKF suitable for non-linear system.

The extended and unscented kalam filters are described in Section 2. The coupled tank along with the non-linearlity of the system is discussed in Section 3. The state equation derived through modification of the state space matrix for simulation purposes is also shown. The computer simulation, experiment results and analysis are outlined in Section 4. Section 5 of Conclusion discusses the experimental results and the conclusions drawn.

\section{Estimation Methods}

The estimation method is widely used in engineering applications such as for the state of a dynamic system, the attitude of a robot, navigation system, etc. The estimate filters used for estimating the state and parameters of a dynamic system are described in this section. EKF is the method of extending the conventional kalman filter for non-linear system. It involves the linearization process of state and measurement equations to update the error covariance.

However UKF has a property that calculates the priori estimation value though UT. Therefore, the linearized error can be disregarded since it is not necessary to linearize the non-linear equation.

\subsection{Extended Kalman Filter}

The EKF based on the conventional kalman solves the non-linearity problem of system by suboptimal. And then it uses Taylor extension to approximate the linearized state and measurement equations.

$$
\begin{aligned}
& x_{k}=f\left(x_{k-1}, k-1\right)+w_{k-1} \\
& z_{k}=h\left(x_{k}, k\right)+v_{k}
\end{aligned}
$$


where the status is $x_{k} \in \square^{n}$; the measurement value is $z_{k} \in \square^{m}$ : the process noise and measurement noise are denoted by $w_{k-1} \sim N\left(0, Q_{k-1}\right)$ and $v_{k-1} \sim N\left(0, R_{k-1}\right)$ respectively; the functions $f, h$ are represents non-linear equation and measuremen equation respectively.

Jacobian elements of the system equation are given by (3), (4)

$$
\begin{aligned}
& {\left[F_{x}(x, k-1)\right]_{i, j}=\frac{\partial f_{i}(x, k-1)}{\partial x_{j}}} \\
& {\left[H_{x}(x, k)\right]_{i, j}=\frac{\partial h_{i}(x, k)}{\partial x_{j}}}
\end{aligned}
$$

The algorithm can be divided into two steps namely the time prediction step and the measurement update step.

Time prediction:

$$
\begin{aligned}
& \hat{x}_{\bar{k}}=f\left(\hat{x}_{k-1}, k-1\right) \\
& P_{\bar{k}}=F_{k} P_{k-1} F_{k}^{T}+Q
\end{aligned}
$$

Measurement update:

$$
\begin{aligned}
& K=P_{\bar{k}} H^{T}\left(H_{k} P_{k} H_{k}^{T}+R_{k}\right) \\
& \hat{x}_{k}=\hat{x}_{\bar{k}}+K\left(z_{k}-H_{k} \hat{x}_{\bar{k}}\right) \\
& P_{k}=(I-K H) P_{\bar{k}}
\end{aligned}
$$

where $\hat{x}$ is the estimate value, the subscript $\bar{k}$ denotes a prior estimation value, subscript $k$ presents a posterior estimation value respectively.

\subsection{Unscented Kalman Filter}

UKF based on UT without the linearization process could effectively be applied to nonlinear systems. It gernerally has a better performance than EKF, because it is not necessary to calculate the jacobian matrix of system equation and then shows the improving approach for non-linear system [11].

The UKF algorithm is as follows;

Initialize with:

$$
\begin{aligned}
& \hat{x}_{0}=E\left[x_{0}\right] \\
& P_{0}=E\left[\left(x_{0}-\hat{x}_{0}\right)\left(x_{0}-\hat{x}_{0}\right)^{T}\right]
\end{aligned}
$$


For $k \in\{1, \ldots, \infty\}$

Calculate sigma points:

$$
\chi_{K-1}^{i}=\left[\hat{x}_{k-1}, \hat{x}_{k-1}+\sqrt{(n+\lambda) P_{k-1}}, \hat{x}_{k-1}-\sqrt{(n+\lambda) P_{k-1}}\right]
$$

Time update:

$$
\begin{gathered}
\chi_{k \mid k-1}^{i}=F\left[\chi_{k-1}^{i}\right] \\
\hat{x}_{k \mid k-1}=\sum_{i=0}^{2 n} w_{i}^{(m)} \chi^{i} k \mid k-1 \\
P_{k \mid k-1}=\sum_{i=0}^{2 n} w_{i}^{(c)}\left[\chi^{i}{ }_{k \mid k-1}-\hat{x}_{k \mid k-1}\right]\left[\chi^{i} k \mid k-1-\hat{x}_{k \mid k-1}\right]^{T} \\
Z_{k \mid k-1}^{i}=H\left[\chi_{k \mid k-1}^{i}\right] \\
\hat{z}_{k \mid k-1}=\sum_{i=0}^{2 n} w_{i}^{(m)} Z_{k \mid k-1}^{i}
\end{gathered}
$$

Measurement update equations:

$$
\begin{gathered}
P_{k \mid k-1}^{z z}=\sum_{i=0}^{2 n} w_{i}^{(c)}\left[Z^{i}{ }_{k \mid k-1}-\hat{z}_{k \mid k-1}\right]\left[Z^{i}{ }_{k \mid k-1}-\hat{z}_{k \mid k-1}\right]^{T} \\
P_{k \mid k-1}^{x z}=\sum_{i=0}^{2 n} w_{i}^{(c)}\left[\chi^{i}{ }_{k \mid k-1}-\hat{x}_{k \mid k-1}\right]\left[Z_{k \mid k-1}^{i}-\hat{z}_{k \mid k-1}\right]^{T} \\
K=P_{k \mid k-1}^{x z}\left(P_{k \mid k-1}^{z z}\right)^{-1} \\
\hat{x}_{k \mid k}=\hat{x}_{k \mid k-1}+K\left(z_{k}-\hat{z}_{k \mid k-1}\right) \\
P_{k \mid k}=P_{k \mid k-1}-K\left(P_{k \mid k-1}^{z z}\right) K^{T}
\end{gathered}
$$

where $P_{k}$ is state error covariance, $P_{k}^{x z}$ is correlation error covariance, $w_{i}^{(m)}, w_{i}^{(c)}$ are the weights.

\section{System and Equipment}

The dynamic system and the state and parameter estimation process are described in this chapter. The dynamic system is setup in order to obtain the system measurement data. The 
equation for the system is derived via mathematical methods. The derived equation is transformed into state space equation form. The dynamic system tested is the coupled tank. The system has second order ordinary differential equation (ODE). The orifice coefficient parameter is important for the stability and control of the system.

\subsection{Coupled Tank}

The coupled tank shown in Figure 1 is manufactured by Quanser innovate. Educate Co. The system is comprised of two tanks, a pump and a water basin. Both tanks has a height of 32 inches with an inside radius of $4.5 \mathrm{~cm}$. The way the tanks are mounted, water is pumped into Tank 1 from a water basin and then water from Tank 1 flows into Tank 2 and then out into the water basin.

The system was constructed to control the pump but is used to deal with parameter estimation in our experiments [12]. The numerical model of Coupled tank is comprised of an input and output function is as follows;

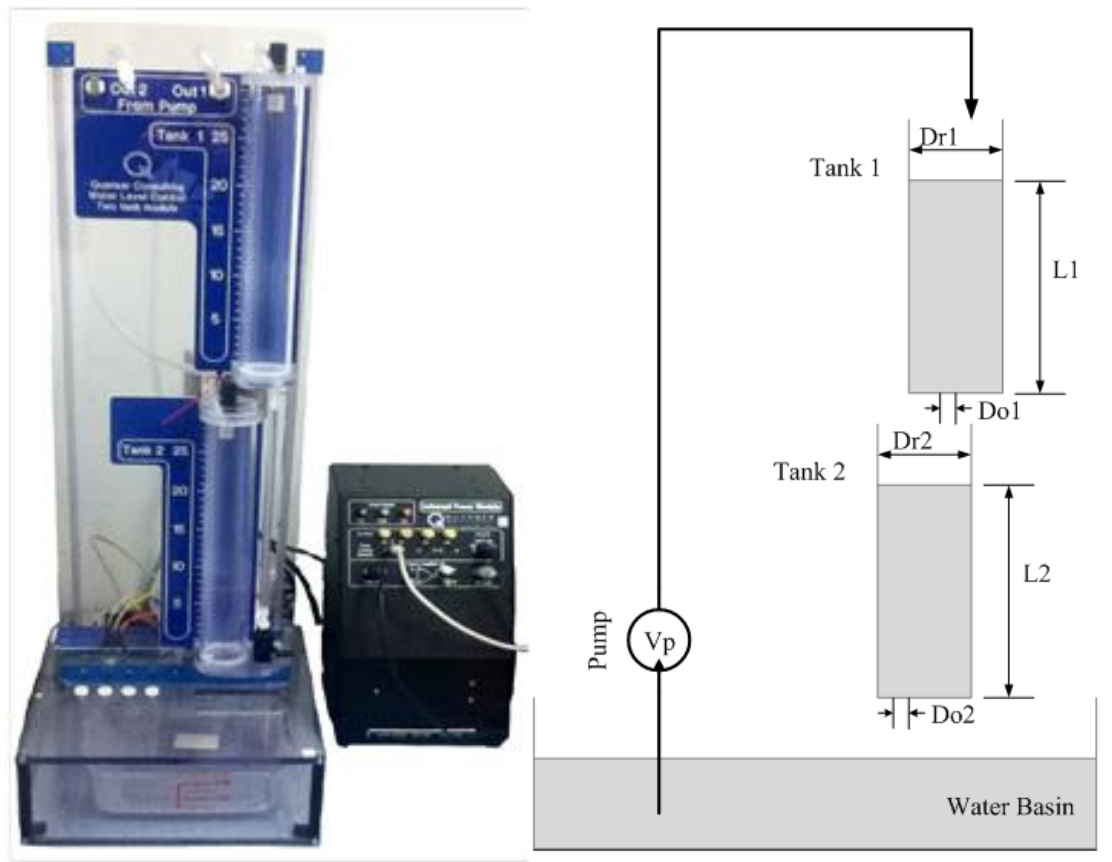

Figure 1. Coupled Tank system at right side and block diagram of coupled tank at left side

$$
\begin{aligned}
& \frac{d h_{1}}{d t}=f\left(h_{1}, q_{i 1}\right) \\
& \frac{d h_{2}}{d t}=f\left(h_{2}, q_{i 2}\right)
\end{aligned}
$$

where $h_{1}$ and $h_{2}$ are the height of tank1 and tank 2, and $q_{i 1}$ and $q_{i 2}$ mean the flowing quantity into tank 1 and tank 2, respectively. The height of tank 1 and 2 could be obtained 
from calculating the difference between inflow and outflow. The outflow quantity of the tanks is given below;

$$
c_{1} \sqrt{h_{1}}, c_{2} \sqrt{h_{2}}
$$

where $c_{1}$ and $c_{2}$ are the orifice coefficients.

The inflow quantity of water in the tank 2 is same as outflow quantity of water in the tank 1. Therefore, the total equation that is including the input from the pump is derived as follows;

$$
\begin{aligned}
& q_{i 1}-c_{1} \sqrt{h_{1}}-A_{1} \frac{d h_{1}}{d t}=0 \\
& c_{1} \sqrt{h_{1}}-c_{2} \sqrt{h_{2}}-A_{2} \frac{d h_{2}}{d t}=0 \\
& q_{i}=k v_{i}
\end{aligned}
$$

where $k$ is [cm/volts] constants, and $v_{i}$ is the pump input, and $A_{1}, A_{2}$ denote the inside areas of tanks. In order to transform the equation adapted the filters, we are defined as $A_{1} \frac{d h_{1}}{d t}=A_{1} h_{1}^{\&}, A_{2} \frac{d h_{2}}{d t}=A_{2} k_{2}^{\&}$. And then it arrangs the either sides of equation from the equations (26) and (27), is given by (29).

$$
\begin{aligned}
& A_{1} k_{1}^{\&}=k v_{i}-c_{1} \sqrt{h_{1}} \\
& A_{2} h_{2}^{\&}=c_{1} \sqrt{h_{1}}-c_{2} \sqrt{h_{2}}
\end{aligned}
$$

The state space matrix form is obtained to apply to the filters.

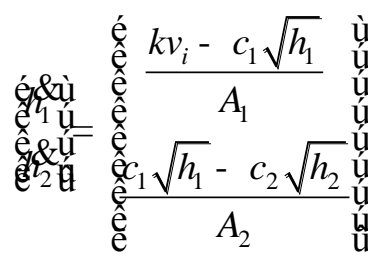

\subsection{Data Acquisition System}

The equipment and specifications to experiment the system are denoted in Table 1 we also assume the following rules. Table 1 lists the equipment and specifications of the experimental setup for the system. The following rules are also observed: In a coupled tank, a fixed amount of water is pumped into Tank 1 and the amount of water in Tank 2 is dependent on the outflow of Tank 1.

This process is continuously repeated until Tank 1 is full. The measurement values are the height of the tanks. In order to measure the data, we set up the data sampling frequency at $100 \mathrm{~Hz}$ with data type at the volts level. Data such as height of the tanks are measured using 
DAQ Board which are transmitted and saved to MATLAB SIMULINK. The calibration of the measuring equipment is based on the value before an operation. The block diagram for measurement process is drawn in Figure 2.

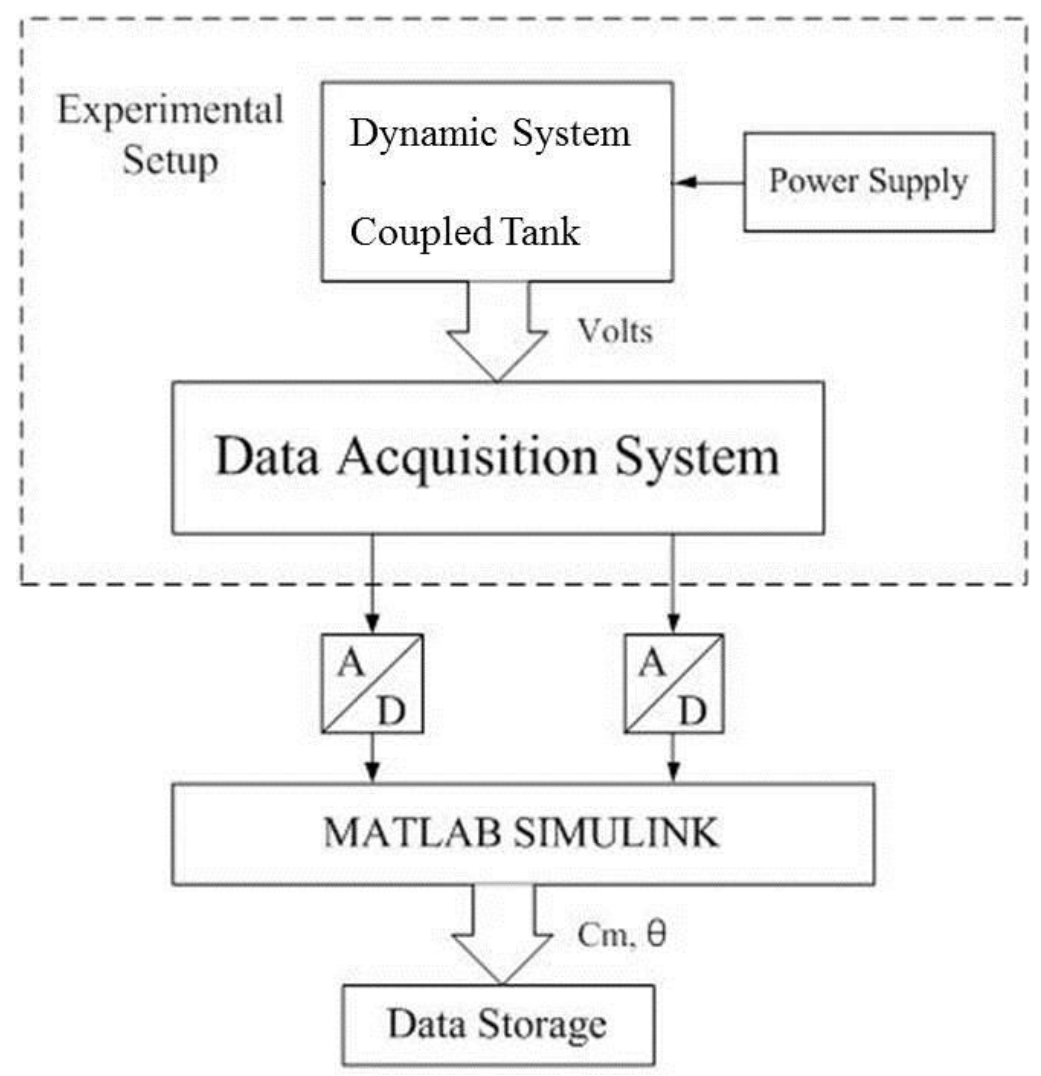

Figure 2. Block diagram for measurement

Table 1. Equipment and Specifications

\begin{tabular}{cc}
\hline Item & Specifications \\
\hline Computer & Regulation $: 0.01 \%$ \\
Hewlett Packard & Ripple $: 200 \mu \mathrm{V}$ \\
6253A & Analog I/O \\
BNC-2120 & Digital I/O \\
Connector Block & Real-time Embedded Controller. \\
NI PXI 8145-RT & Processor : 266MHz low-power Intel Pentium MMX \\
& Interface : Serial, CAN, MXI-3 chassis expansion \\
\hline NI PCI 6064E & Resolution : 12,16 bit \\
DAQ Board & Channel : $500 \mathrm{ks} / \mathrm{s}$ \\
NI PXI & Multichannel : $250 \mathrm{ks} / \mathrm{s}$ \\
1031-Chassis & Accuracy : $\pm 25 \mathrm{ppm}$ \\
Initial values & Maximum Clock : $250 \mathrm{ps}$ \\
& Coupled Tank \\
& Height of Tank $1: 0$ \\
& Height of Tank $2: 0$ \\
\hline
\end{tabular}




\subsection{Parameter Table}

The parameter of the dynamic system is represented in Table 2. Most of parameter is directly measured value or the initial value when it is constructed.

Table 2. Parameter of Coupled tank

\begin{tabular}{c|c|c|c}
\hline Description & Sym. & Val. & Unit \\
\hline Cross-sectional areas of tank 1 & $A_{1}$ & 15.5179 & $\mathrm{~cm}^{2}$ \\
\hline Cross-sectional areas of tank 2 & $A_{2}$ & 15.5179 & $\mathrm{~cm}^{2}$ \\
\hline Pumping rate & $q_{i}$ & 5 & Volts \\
\hline Flow constant & $k$ & 6 & $\mathrm{~cm} /$ Volts \\
\hline Orifice coefficient of tank 1 & $c_{1}$ & 5 & \\
\hline Orifice coefficient of tank 2 & $c_{2}$ & 5 & \\
\hline
\end{tabular}

\section{Experiments}

The state and parameter estimation performance is based on MATLAB, EKF and UKF. The result of estimating the parameters of the two orifice coefficients of the tanks are analyzed. Computer simulation and then experimental tests were done to estimate the parameters.

The estimation of the state and parameter is performed based on MATLAB for EKF, UKF. Two orifice coefficients of the system are estimated. It is analyzed as the results of the parameter estimation. The EKF and UKF were then applied to the real system wherein the measurement noise was obtained and determined by measuring the noise when the system was stopped.

\subsection{State Estimation}

The state and parameter estimation result is shown in Figure 3 The estimation error at the initial part of the process is emphasized as shown. The algorithms for EKF and UKF estimate the state value on the measurement data. The measurement error in Tank 1 occurred when the pumped water dropped into the tank. In Figure 2, each solid line is the measurement data, the results are represented as solid-solid line for EKF and solid-dot line for UKF.

\subsection{Parameter Estimation}

The unexpected state error happened due to the initial water pressure dropped from the pump. According to that error, the estimation value that is the orifice coefficient initially increased but stabilized afterward through recursive update. 

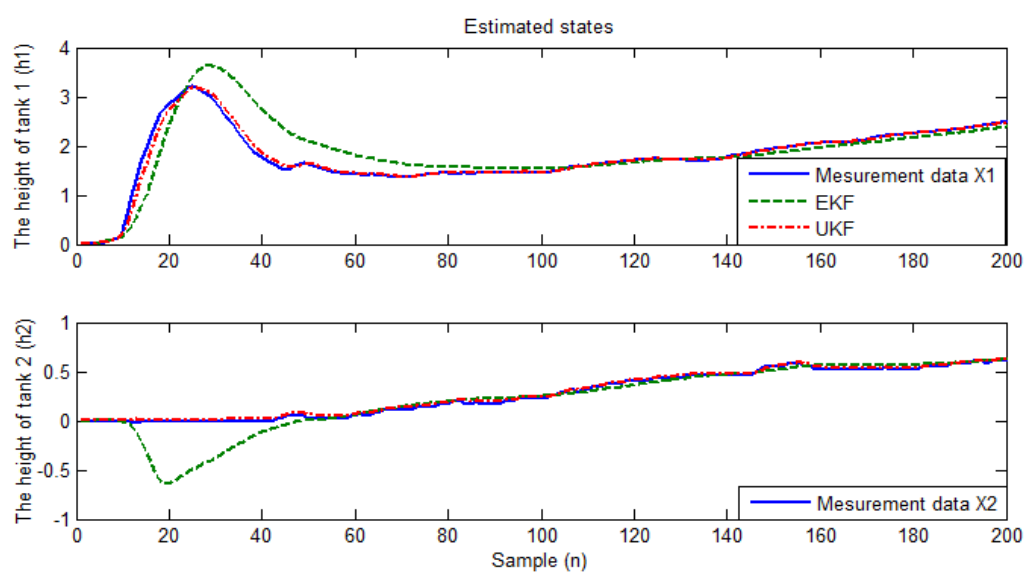

Figure 3. Result of State Estimation for Coupled Tank
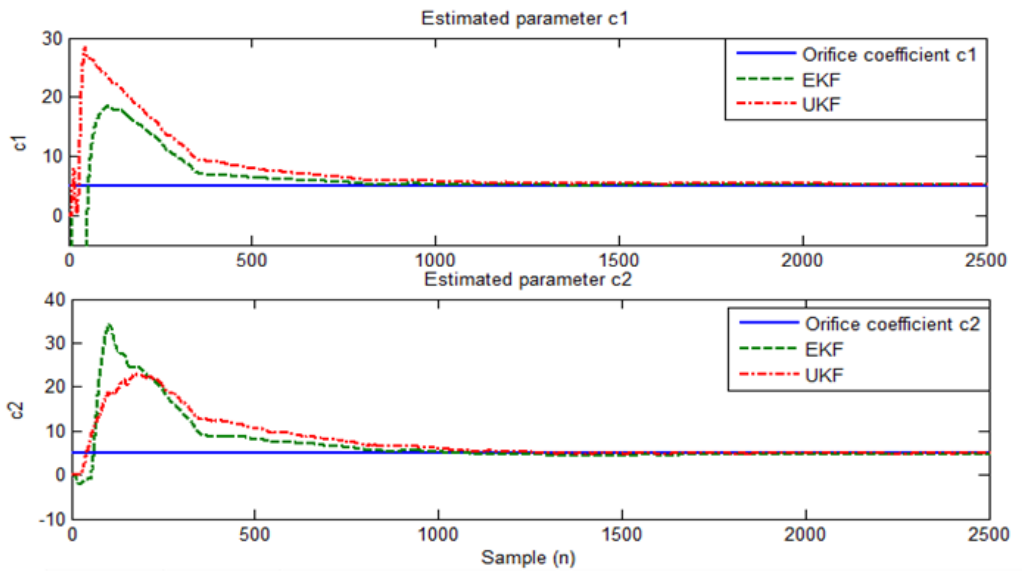

\section{Figure 4. Result of Estimation for Orifice Coefficient of Tank 1 and Tank 2}

\subsection{Comparison}

The estimation error and results are analyzed, calculated and compared using mathematical methods. The estimation performance is analyzed using objective results of the processing time of the filters.

Accuracy :Based on the computer simulation and experimental results of EKF and UKF from the previous section, the performance of each is analyzed through their RMSE. Table 3 denoted the result of the parameter estimation of coupled tank system. where value is the estimated value, RMS is calculated after the converged time at reference value.

Table 3. Result of Parameter Estimation for Coupled Tank

\begin{tabular}{|c|c|c|c|c|c|}
\hline \multirow{2}{*}{\multicolumn{2}{|c|}{ Unknown parameter }} & \multicolumn{2}{|c|}{$\mathrm{EKF}_{(\mathrm{RMS})}$} & \multicolumn{2}{|c|}{$\mathrm{UKF}_{(\mathrm{RMS})}$} \\
\hline & & Value & Error & Value & Error \\
\hline$c 1$ & 5.00 & 5.0946 & 0.0946 & 5.0245 & 0.0245 \\
\hline$c 2$ & 5.00 & 5.0522 & 0.0522 & 5.0123 & 0.0123 \\
\hline
\end{tabular}

The experiment results for Couple Tank is shown in Table 4. 
Table 4. Result of Parameter Estimation for Coupled Tank

\begin{tabular}{|c|c|c|c|c|c|}
\hline \multirow{2}{*}{\multicolumn{2}{|c|}{ Unknown parameter }} & \multicolumn{2}{|c|}{$\mathrm{EKF}_{(\mathrm{RMS})}$} & \multicolumn{2}{|c|}{$\mathrm{UKF}_{(\mathrm{RMS})}$} \\
\hline & & Value & Error & Value & Error \\
\hline$c 1$ & 5.00 & 5.1285 & 0.1285 & 5.1201 & 0.1201 \\
\hline$c 2$ & 5.00 & 4.6336 & 0.3664 & 4.9615 & 0.0385 \\
\hline
\end{tabular}

Estimating time:Although the estimating time varies according to the simulation environment and the kinds of trajectory, the results are trustworthy because the estimation of all filter is implemented using the same lab equipments and data set

Table 5. Estimating Time of filters

\begin{tabular}{cccc}
\hline \multicolumn{2}{c}{ Dynamic System } & EKF $_{(\mathrm{sec})}$ & $\mathrm{UKF}_{(\mathrm{sec})}$ \\
\hline \multirow{2}{*}{ Coupled Tank } & Com. Simulation & 0.452 & 1.656 \\
& Exp. Simulation & 0.602 & 2.249 \\
\hline
\end{tabular}

In Table 5, EKF requires $0.452 \mathrm{sec}$ for estimating the state and parameter and UKF needs $1.656 \mathrm{sec}$ on simulation. On experiment, the estimating time of UKF takes more time than EKF, 3.74 times.

\section{Conclusion}

For this research, models were derived, dynamic systems were constructed and the computer simulation and experimental tests were performed. The measurement of data systems were collected through NI DAQ Board and linked to MATLAB Simulink.

The state and parameters are estimated in order to analyze the performance of extended kalman filter and unscented kalman filter. The orifice coefficients of a coupled tank that affects system stability were estimated for this research.

As the results of the accuracy of estimation, UKF shows an outstanding performance for estimating the state and parameter. On the other hand, the results of EKF estimation have a lot of estimation error because of linearization of state equation.

The last test done was to check how long the filters need to estimate the state and parameters. It showed that the UKF required as much as 3.74 times it took EKF. Computer simulations also showed that both EKF and UKF performed excellently when used to estimate the state and parameters. However, the estimation performance of UKF gave the better result over EKF which makes it suitable for real-time estimation. This research was able to verify the accuracy and reliability of UKF.

\section{Acknowledgements}

This work was supported by the National Research Foundation of Korea (NRF) grant funded by the Korea government (MEST) (No. 2012038978) and (No. 20120002434)

\section{References}

[1] W. J. Sung, S. O. Choi and K. H. You, "TDoA based UGV Localization using Adaptive Kalman Filter Algorithm", International Journal of Control and Automation, vol. 2, (2009), pp. 1-10.

[2] D. K. Kim, J. H. Ha and K. H. You, "Adaptive Extended Kalman Filter Based Geolocation Using TDOA/FDOA", International Journal of Control and Automation, vol. 4, (2010), pp. 49-58. 
[3] D. T. Lee, M. Morf and B. Friedkander, "Recursive least - squares ladder estimation algorithm", IEEE Trans. Acoust Speech. Signal Processing, vol. 29, (1981), pp. 627-641.

[4] J. Swever, C. S. Gansenman, D. B. Tukel and J. De schutter, "Optimal Robot Excitation and Identification", IEEE Transactions on Robotics and Automation, vol. 13, no. 5, (1997) October, pp. 730-740.

[5] S. -K. Oh, W. Pedrycz, S. -B. Rho and T. -C. Ahn, "Parameter estimation of fuzzy controller and its application to inverted pendulum", Engineering Applications of Artificial Intelligence, vol. 17, (2004), pp. 37-60.

[6] J. W. Park, Y. S. Ha and W. Z. Chung, "Kalman Filtering based Adaptive Frequency Domain Channel Estimation with Low Pilot Overhead for OFDM Systems", International Journal of Control and Automation, vol. 5, (2012), pp. 107-114.

[7] S. Aksoy, A. Mühürcü and H. Kızmaz, "State and Parameter Estimation in Induction Motor Using the Extended Kalman Filtering Algorithm", Modern Electric Power Systems 2010, Wroclaw, Poland, (2010).

[8] V. Panuska, "A New Form of the Extended Kalman Filter for Parameter Estimation in Linear Systems with Correlated Noise", IEEE Transactions on Automatic Control, vol. AC-25, no. 2, (1980).

[9] E. Blanchard, A. Sandu and C. Sandu, "Parameter Estimation Method using an Extended Kalman Filter", Proceedings of the Joint North America, Asia-Pacific ISTVS Conference and Annual Meeting of Japanese Society for Terramechanics, (2007).

[10] M. Zheng, K. Ikeda and T. Shimomura, "Estimation of Rotary Inverted Pendulum by using the Unscented Kalman Filter-estimation of the initial state", SICE Annual Conference 2007, (2007).

[11] S. Julier and J. Uhlmann, "A new extension of the Kalman filter to nonlinear systems", Proceedings of the 1997 SPIE AeroSense Symposium, SPIE, vol. 3068, (1997), pp. 182-193.

[12] http://quanser.com/english/html/products/fs_product_challenge.asp?lang_code=english\&pcat_code=expspe\&prod_code $=\mathrm{S} 13$-tanks\&tmpl $=1$.

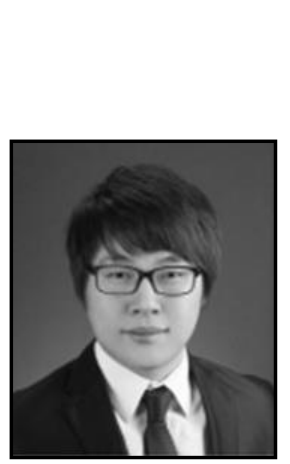

\section{Authors}

Ji-hoon Seung was born in Jeonju, South Korea. He received his B.S and M.S degree in 2010 and 2013 from Chonbuk Nation University (South Korea), all in electronic engineering. He was a Visiting Students at Texas A\&M University from 2011 to 2012. His research interests are in the fields of estimation methods, navigation and sensor fusion.

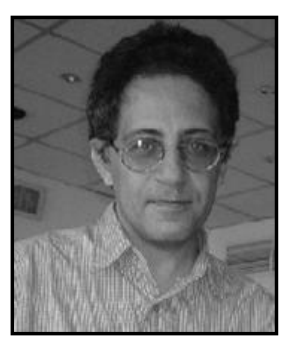

Amir Atiya F. was born in Cairo, Egypt. He received his B.S. degree in 1982 from Cairo University (Egypt), and the M.S. and Ph.D. degrees in 1986 and 1991 from Caltech, Pasadena, CA, all in electrical engineering. He held positions in academia, as well as several positions in financial firms. From 1997 to 2001 he was a Visiting Associate at Caltech. On leave from Cairo University, he recently held research positions in the firms Simplex Risk Management, Hong Kong, Countrywide Corporation in Los Angeles, and Dunn Capital Management, Florida. Currently, he is a Research Scientist with Veros Systems, Texas. His research interests are in the fields of neural networks, signal processing, optimization theory, and pattern recognition. 


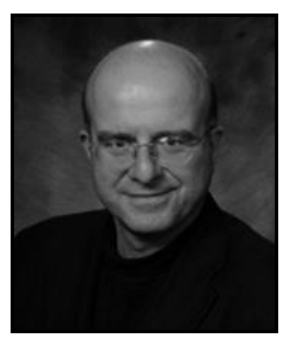

Alexander G. Parlos received the B.S. degree in nuclear engineering from Texas A\&M University, College Station, and the S.M. degree in nuclear engineering, the S.M. degree in mechanical engineering, and the Sc.D. degree in automatic control and systems engineering, all from the Massachusetts Institute of Technology (MIT), Cambridge. $\mathrm{He}$ is currently a Professor of Mechanical Engineering at Texas A\&M University, holding joint appointments with the Department of Nuclear Engineering, and, by courtesy, the Department of Electrical Engineering. His applied research interests include intelligent sensor networks for lifecycle condition assessment (fault diagnosis), end-of-life prediction (prognosis), and reconfigurable control.

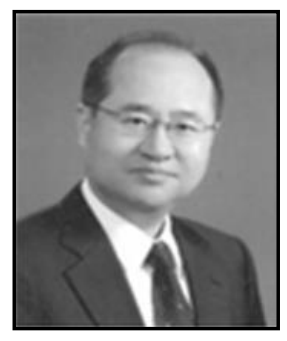

Kilto Chong received the B.S degree from Oregon State University (USA) in Mechanical Engineering, and the M.S degree from Georgia Institute of Technology (USA) in Mechanical Engineering, and the Ph. D degree from Texas A\&M University (USA) in Mechanical Engineering. Currently, he is professor and dean of Electronics Engineering Division in Chonbuk National University, president of Electronics and IT New Technologies Research Center in Chonbuk National University. His research interests fields are Time-Delay, Robotics, Artificial Intelligence and Sensor Networks. 